\title{
Ecofriendly, Economic Surrogative for Xylene
}

\author{
Janani Ilango ${ }^{1}$, M.Devi $^{2}$, D.Vijayalakshmi ${ }^{3}$ \\ ${ }^{1}$ Post graduate student, Department of Oral Pathology,Adhiparasakthi Dental College and Hospital, \\ Melmaruvathur, Tamil Nadu, India \\ ${ }^{2}$ Professor,Department of Oral Pathology,Adhiparasakthi Dental College and Hospital,Melmaruvathur, Tamil \\ Nadu, India \\ ${ }^{3}$ Professor,Department of Oral Pathology,Adhiparasakthi Dental College and Hospital,Melmaruvathur, Tamil \\ Nadu, India
}

\begin{abstract}
Background: Xylene is an aromatic hydrocarbon, mostly used as a clearing agent during tissue processing and as a de-waxing agent during histopathological staining. The biohazardous nature ofxylene, makes it a potential occupational hazard for histopathological technicians. Exposure to xylene can occur via inhalation, ingestion or direct contact with skin, eyes etc. Exposure and handling sections of xylene is maximum during dewaxing of sections. These have led to the question of a substitute agent which is less toxic and safer.

Aim: To assess the efficacy of 1.5\% of dishwashing liquid and 1.5\% of detergent liquid as a deparaffinizing agent for $H \& E$ staining technique.

Objective:To utilize eco-friendly, economic substitute for xylene.

Materials and Methods: Using 20 paraffin embedded tissue blocks, three sections were prepared and considered into 3 groups as Group A, Group B, Group C. Group A was stained with conventional H\&E method, Group B was stained using $1.5 \%$ of dishwashing liquid as a deparaffinizing agent and Group $C$ was stained using $1.5 \%$ of detergent liquid as a deparaffinizing agent.

Statistical Analysis: ANOVA test was used to calculate the test of significance.

Result and Conclusion:1.5\% of dishwashing liquid and 1.5\% of detergent liquid are comparatively an effective alternative for xylene thus acting as anecofriendly, economic surrogative for xylene.

Keywords:xylene, dishwashing liquid, detergent liquid
\end{abstract}

\section{Introduction}

Xylol or dimethylbenzene also called as xylene, is an aromatic synthetic hydrocarbon that plays a vital role in pathological laboratory formany years. It is availablenaturally in the form of coal tar and petroleum, obtained its name from crude wood spirit. ${ }^{1}$ It is colorless, flammable liquid or gas with a sweet odour. Xylene is being used as a clearing agent in tissue processing where it causesmaximum displacement of alcohol and makes the tissue transparent thus enhancing paraffin infiltration and acts as a deparaffinizing agent in staining procedure. ${ }^{2}$ Although it is extremely useful, when exposed, it leads to health hazards to almost all parts of the bodyranging from nausea, vomiting to death. Current Occupational Safety and Health Administration permissible limit for exposure to xylene is $100 \mathrm{ppm}$ as an 8-hour time-weighted average (TWA) concentration. ${ }^{3}$ Limonene reagents, aliphatic hydrocarbon mixtures, aromatic hydrocarbon mixtures, and mineral oil mixtures were used as alternatives for xylene in tissue processing as clearing agent. ${ }^{4}$ But peak exposure takes placeduring dewaxing of sections. Long term exposure leads to permanent disability caused by diminution of mitochondrial adenosine triphosphate in the affected cells. ${ }^{5}$ Hence, the present study is intended to replace xylene with nonbiohazardous agents like dish washing liquid and detergent liquid.

Aim: The aim of the present study isto assess the efficacy of $1.5 \%$ of dishwashing liquid and $1.5 \%$ of detergent liquid as a deparaffinizing agent for H\&E staining technique and to utilize eco-friendly, economic substitute for xylene

\section{Materials and Methods}

Twenty paraffin embedded tissue blocks from our department were obtained. Three sections of 4 to 5 microns thickness were prepared fromeach block. One section was stained with Conventional H\&Emethod where xylene was used as deparaffinizing agent. The other two sections were stained with Xylene FreeHematoxylin and Eosin ( $\mathrm{H}$ and E), where 1.5\% Dishwashing liquid (1.5mL Vim dish washing solutionin $98.5 \mathrm{~mL}$ distilled water) and $1.5 \%$ of Detergent liquid (1.5mL Surf excel detergent washing solutionin $98.5 \mathrm{~mL}$ distilled water) were used as deparaffinising agent.The three sections were categorized as: 
Group A:Stained using 1.5\% of detergent liquid as a deparaffinizing agent (Fig. 1)

Group B: Stained using $1.5 \%$ of dishwashing liquid as a deparaffinizing agent (Fig. 2)

Group C: Stained using xylene as a deparaffinizing agent (Fig. 3)

Each section were assessed separately and scored accordingly by three different pathologists. The parameters for scoring included nuclear staining, cytoplasmic staining (adequate $=$ score 1 , inadequate $=$ score 0 ), uniformity, clarity, crispness $($ present $=$ score 1, absent $=$ score 0$)$

The protocol followed for group A, group B and group C are given in tables $1 \& 2$ respectively

\section{Deparaffinization}

Table 1: Routine $\mathrm{H}$ and $\mathrm{E}$ staining using xylene asdewaxing agent

Xylene I - 5 min

Xylene II - 5 min

$90 \%$ alcohol $-5 \mathrm{~min}$

$70 \%$ alcohol - 5 min

Water wash - $10 \mathrm{~min}$

Nuclearstaining

Harris hematoxylin - 8 min

Water wash - 2 min

Differentiation

$1 \%$ acid alcohol $1 \mathrm{dip}$

Bluing

Tap water wash - $10 \mathrm{~min}$

Cytoplasmicstaining

$1 \%$ eosin - 2 min

Dehydration

$70 \%$ alcohol - $30 \mathrm{~s}$

$90 \%$ alcohol $-30 \mathrm{~s}$

Xylene - 5 min

Approximate time: $55-60 \mathrm{~min}$

Table 2: Xylene free staining using 1.5\% Dishwashing liquid (DWL)\& 1.5\% Detergent liquid (DL) as

Deparaffinization

dewaxing agent

$1.5 \%$ DWL I \& DL I at $90^{\circ} \mathrm{C}-2 \mathrm{~min}$

$1.5 \%$ DWL II\& DL II at $90^{\circ} \mathrm{C}-2 \mathrm{~min}$

Distilled waterI at $90^{\circ} \mathrm{C}-30 \mathrm{~s}$

Distilled waterII at $90^{\circ} \mathrm{C}-30 \mathrm{~s}$

Distilled water at $45^{\circ} \mathrm{C}-30 \mathrm{~s}$

Distilled water at room temperature - $30 \mathrm{~s}$

Nuclear staining

Harris hematoxylin - 8 min

Water wash - 2 min

Differentiation

$1 \%$ acid alcohol $-1 \mathrm{dip}$

Bluing

Tap water wash - $10 \mathrm{~min}$

Cytoplasmic staining

$1 \%$ eosin - 2 min

Water wash - 1 min

Dehydration

Overdrying at $60^{\circ} \mathrm{C}-5 \mathrm{~min}$

Approximate time: $35-40$ min

\section{Statistics}

Total score for each criteria was calculated by three pathologists. ANOVA test was done using SPSS 17 software to calculate the test of significance $(\mathrm{P} \leq 0.05)$ 


\section{Results}

It was noted that the results were statistically insignificant for all criteria with a slight increase in the mean values for group A \& group B when compared to group C in 4 out of 5 criteria.(Table 3 to 7 )

TABLES

Table 3:Clarity of Staining

\begin{tabular}{|l|l|l|l|l|}
\hline Observer & Group A & Group B & Group C & P Value \\
\hline Observer 1 & 0.75 & 0.7 & 0.8 & 0.77 \\
\hline Observer 2 & 0.8 & 0.7 & 0.75 & 0.77 \\
\hline Observer 3 & 0.75 & 0.8 & 0.8 & 0.91 \\
\hline
\end{tabular}

Table 4:Uniformity of Staining

\begin{tabular}{|l|l|l|l|l|}
\hline Observer & Group A & Group B & Group C & P Value \\
\hline Observer 1 & 0.95 & 0.9 & 0.9 & 0.81 \\
\hline Observer 2 & 0.85 & 0.7 & 0.8 & 0.52 \\
\hline Observer 3 & 0.95 & 0.9 & 0.8 & 0.33 \\
\hline
\end{tabular}

Table 5:Crispness of Staining

\begin{tabular}{|l|l|l|l|l|}
\hline Observer & Group A & Group B & Group C & P Value \\
\hline Observer 1 & 0.7 & 0.7 & 0.7 & 0.99 \\
\hline Observer 2 & 0.75 & 0.75 & 0.7 & 0.91 \\
\hline Observer 3 & 0.6 & 0.7 & 0.7 & 0.75 \\
\hline
\end{tabular}

Table 6:Nuclear Staining

\begin{tabular}{|l|l|l|l|l|}
\hline Observer & Group A & Group B & Group C & P Value \\
\hline Observer 1 & 1 & 1 & 0.9 & 0.13 \\
\hline Observer 2 & 0.95 & 0.95 & 0.9 & 0.77 \\
\hline Observer 3 & 0.9 & 1 & 0.9 & 0.35 \\
\hline
\end{tabular}

Table 7:Cytoplasmic Staining

\begin{tabular}{|l|l|l|l|l|}
\hline Observer & Group A & Group B & Group C & P Value \\
\hline Observer 1 & 1 & 1 & 0.9 & 0.13 \\
\hline Observer 2 & 0.9 & 1 & 1 & 0.13 \\
\hline Observer 3 & 0.9 & 0.95 & 1 & 0.35 \\
\hline
\end{tabular}

Figure1:H\&E stained section using $1.5 \%$ of detergent liquid as a deparaffinizing agent



Figure 2:H\&E stained section using $1.5 \%$ of dish wash liquid as a deparaffinizing agent

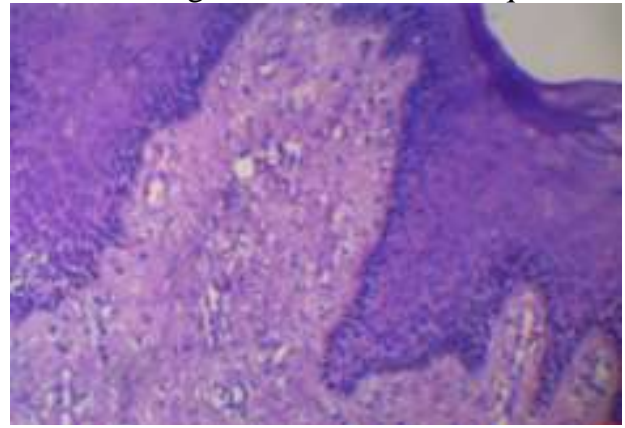


Figure 3:H\&E stained section using xylene as a deparaffinizing agent

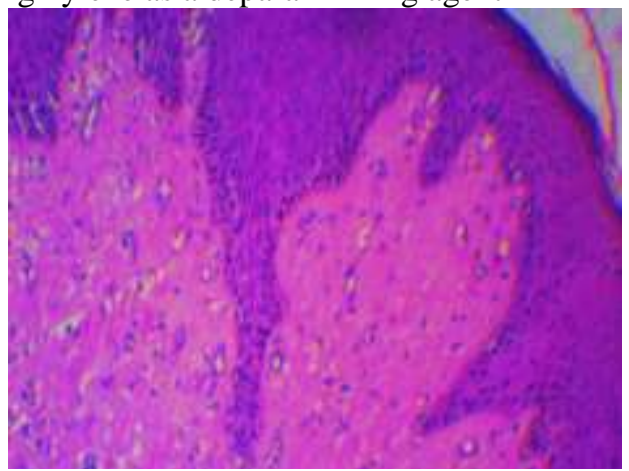

\section{Discussion}

The knowledge of toxicity of xylene is known by many pathologists and lab technicians.Inspite of this it is widely being used in laboratory without any safety measures and standardized method for disposal. ${ }^{6}$ Considering the hazardous nature of the xylene, many substitutes were tried in the past and our study was the first to use detergent liquid as an alternative to xylene in the process of deparaffinization.

The dish wash liquid and the detergent liquid are highly foaming mixture of surfactants, made up of alkylbenzenesulfonates, which as a solvent property that helpsin dissolving wax. ${ }^{7}$ The principle behind this is that the surfactant property along with the high temperature of 90 degree Celsius reduces the surface tension, thus helps in deparaffinizing the section. ${ }^{8}$

Compared to xylene, both detergent liquid\&dishwash liquid are economic, biosafe and readily available. The main constituents of both the liquids are sodium lauryl sulfate, sodium dodecyl benzene sulfonate, cocamidopropylbetaine and nonionic surfactants. The concentration of these components are well analysed by the manufacturer. ${ }^{9}$ In this study, we have used dish wash liquid and the detergent liquid in a dilution of $1.5 \%$, hence there are very minimal chances of toxicity to the laboratory personnel.

In this study, even though the results were statistically insignificant, it implies that the efficacy of dish wash liquid and detergent liquid is equal to that of xylene. The mean values of the observers for uniformity of staining, crispness of staining and nuclear staining shows higher end for xylene free staining. The mean value of one observer for clarity of staining and cytoplasmic staining shows higher end for xylene free staining. Thus the results of the present study is equally effective without compromising the staining quality.

\section{Conclusion}

Considering the safety measures and for the healthy laboratory environment, economic surrogative for xylene such as detergent liquid and dishwash liquid can be used as a deparaffinizing agent.

\section{Reference}

[1] Ananthaneni A, Namala S, Guduru VS, Ramprasad VV, Ramisetty SD, Udayashankar U, Naik KK. Efficacy of 1.5\% dish washing solution and $95 \%$ lemon water in substituting perilous xylene as a deparaffinizing agent for routine $\mathrm{h}$ and e staining procedure: a short study. Scientifica. 2014 Mar 31;2014.

[2] Ba Bancroft JD, Gamble M. Theory and Practice of HistologicTechniques. 6th ed. Philadalphia, USA: Churchill LivingstoneElsevier; 2011.

[3] Kandyala R, Raghavendra SP, Rajasekharan ST. Xylene: An overview of its health hazards and preventive measures. Journal of oral and maxillofacial pathology. $2010 \mathrm{Jan}$ 1;14(1):1.

[4] Ramulu S, Koneru A, Ravikumar S, Sharma P, Ramesh DV, Patil R. Liquid dish washing soap: An excellent substitute for xylene and alcohol in hematoxylin and eosin staining procedure. Journal of Orofacial Sciences. 2012 Jan 1;4(1):37.

[5] Buesa RJ, Peshkov MV. Histology without xylene. Annals of diagnostic pathology. 2009 Aug 31;13(4):246-56.

[6] Negi A, Puri A, Gupta R, Chauhan I, Nangia R, Sachdeva A. Biosafe alternative to xylene: A comparative study. Journal of Oral and Maxillofacial Pathology. 2013 Sep 1;17(3):363.

[7] Falkeholm L, Grant CA, Magnusson A, Möller E. Xylene-free method for histological preparation: a multicentre evaluation. Laboratory investigation. 2001 Sep 1;81(9):1213-21.

[8] Henwood AF, Prasad L, Bourke VM. The application of heated detergent dewaxing and rehydration to techniques for the demonstration of fungi: a comparison to routine xylene-alcohol dewaxing. Journal of Histotechnology. 2013 Jun 1;36(2):45-50.

[9] Ankle MR, Joshi PS. A study to evaluate the efficacy of xylene-free hematoxylin and eosin staining procedure as compared to the conventional hematoxylin and eosin staining: An experimental study. Journal of Oral and Maxillofacial Pathology. 2011 May $1 ; 15(2): 161$. 